\title{
Retracted: Medication Non-Adherence among Patients with Heart Failure
}

Zahid U. Rehman $^{1}$, Arsalan K. Siddiqui ${ }^{2}$, Musa Karim ${ }^{1}$, Haris Majeed ${ }^{3}$, Muhammad Hashim 3

1. Miscellaneous, National Institute of Cardiovascular Diseases, Karachi, PAK 2. Miscellaneous, Aga Khan University Medical College, Aga Khan University Hospital, Karachi, PAK 3. Cardiology, National Institute of Cardiovascular Diseases, Karachi, PAK

$\square$ Corresponding author: Musa Karim, mkarim.nicvd@gmail.com

Disclosures can be found in Additional Information at the end of the article

Corresponding author: Musa Karim

1. Miscellaneous, National Institute of Cardiovascular Diseases, Karachi, PAK 2. Miscellaneous, Aga Khan University Medical College, Aga Khan University Hospital, Karachi, PAK 3. Miscellaneous, National Institute of Cardiovascular Diseases, Karachi, PAK 4. Cardiology, National Institute of Cardiovascular Diseases, Karachi, PAK 5. Cardiology, National Institute of Cardiovascular Diseases, Karachi, PAK

How to cite this retraction

Rehman Z U, Siddiqui A K, Karim M, et al. (November 05, 2019) Retraction: Medication Non-Adherence among Patients with Heart Failure. Cureus 11(11): r17. doi:10.7759/cureus.r17

\section{Retraction Notice}

This article has been retracted due to the authors' unlicensed use of The Morisky Medication Adherence Scale, otherwise known as the Morisky Scale (MMAS-8), which was copyrighted in 2006. Additionally, the scoring and coding of the MMAS-8 is incorrect, thus invalidating the results. 